\title{
Imaging of the Choledocal Cystic Dilatation in Infants and Children
}

\author{
M. Ouha ${ }^{1 *}$, K. Zitouni ${ }^{1}$, B. Zouita ${ }^{1}$, D. Basraoui H. Jalal ${ }^{1}$
}

\author{
${ }^{1}$ Department of Pediatric Radiology, Mother and Child Hospital, University Hospital Mohammed VI, Marrakech, Morocco \\ DOI: $10.36347 /$ sasjm.2021.v07i04.009 \\ | Received: 18.03.2021 | Accepted: 24.04.2021 | Published: 29.04.2021 \\ *Corresponding author: M. Ouha
}

Abstract

Original Research Article

Purpose: The choledochal cyst is a rare surgical entity due to an anomaly of the bilio-pancreatic junction, which manifests itself mainly during childhood and often revealed by a complication. Imaging plays an important role in the positive diagnosis, allows to analyze the malformation of the main bile duct and to classify it according to the classification of Todani. The aim of our work is to show the contribution of imaging in the diagnosis of this disease in Pediatric Population and its complications. Methods and Materials: This is a retrospective descriptive study of 19 case reports carried out between 2013 and 2020 in the Pediatric radiology and Child Surgery departments at the mother and child hospital at the University Hospital MOHAMED VI. Results: The average age of our patients was 5 years, predominantly female. Abdominal pain was the most common clinical sign. All patients were examined by ultrasound, 11 cases had abdominal CT with injection of contrast agent, while a bili-MRI was performed in 7 patients. These examinations allowed us to objectify a cystic dilatation of the bile duct classified type I according to Todani in $80 \%$ of cases, and a type IVa in $20 \%$ of patients. Treatment consisted of a resection of the cyst with hepaticojejunal anastomosis associated with cholecystectomy, the postoperative outcome was good. Conclusion: Medical imaging plays an essential role in the diagnosis of cystic dilatations of the bile duct. Bili-MRI is the exam of choice that allows accurate biliary mapping, avoiding the use of invasive opacifications.

Keywords: The choledochal cyst, Child Surgery, Medical imaging.

Copyright $\odot 2021$ The Author(s): This is an open-access article distributed under the terms of the Creative Commons Attribution 4.0 International License (CC BY-NC 4.0) which permits unrestricted use, distribution, and reproduction in any medium for non-commercial use provided the original author and source are credited.

\section{INTRODUCTION}

Cystic dilations of the bile ducts are congenital malformations, described for the first time in 1723 by VATER, characterized by a cystic dilatation which connects with biliary tracts. This disease includes several types, depending on the localization, the shape and distribution of the cyst. This pathology manifests itself mainly during childhood and often revealed by a complication. The diagnosis is essentially based on ultrasound and Magnetic Resonance Cholangiopancreatography.

By reporting a series of 19 children, the aim of this work is to show the contribution of imaging in the diagnosis of this condition and its complications in children and infants as well.

\section{MATERIAL AND METHODS}

We conducted a retrospective descriptive study of 19 case reports carried out between 2013 and 2020 in the Pediatric radiology and Child Surgery departments at the mother and child hospital at the University Hospital MOHAMED VI. The average age of our patients was 5 years, mostly female $(\mathrm{n}=12)$. Abdominal pain was the most common clinical sign. All patients were scanned by ultrasound, 11 cases underwent abdominal CT with contrast agent injection, while a Magnetic Resonance Cholangiopancreatography (MRCP) was performed in 7 patients. These examinations allowed us to objectify a cystic dilatation of the bile duct classified type I according to Todani in $80 \%$ of cases, and a type IVa in $20 \%$ of the patients. Treatment consisted of resection of the cyst with hepato-jejunal anastomosis associated with cholecystectomy with good postoperative outcome. The anathomopathological study of the cysts showed no evidence of malignancy in all children, an aspect of hepatic cirrhosis was observed in one case.

\section{DISCUSSION}

Bile duct cyst is a rare condition. Its incidence is $1 / 100,000$ to $1 / 200,000$ births, with a clear predominance in the female sex. Usually this pathology is diagnosed before the age of 10 years. Nevertheless, $20-50 \%$ of cases are discovered in adulthood in Western countries [1]. 
From an etiopathogenic point of view, it results from an abnormality of the bilio-pancreatic junction where the Wirsung unites with the bile duct upstream of the sphincter thus allowing the reflux of pancreatic secretions into the bile duct, which leads to an incomplete and intermittent obstruction of the low bile duct, explaining the progressive and late character of clinical signs [2].

The clinical presentation is characterized by the classic triad: abdominal pain, jaundice, and a mass of the right hypochondrium. This symptomatology is observed in only one third of cases. Choledocal cysts can be complicated by pancreatitis, cholangitis or peritonitis by spontaneous rupture, but it can also remain asymptomatic, so the discovery is fortuitous during a medical imaging examination [3].

From the anatomical point of view, the first description of ductal dilatation of the biliary tree was mad by Vater in 1723. In 1959, Alonso- Lej proposed the first classification of these anomalies, currently; however, Todani's classification is the most used, which describe five types according to the seat, extent and type of dilatation of the bile ducts [4].

The diagnosis of cystic dilatation of biliary ducts is currently based on non-invasive imaging methods: ultrasound, CT and MR cholangiography.

Diagnosis can be done prenatally by ultrasound or MRI which allows the detection of the formation of the hepatic hilum suggestive of a biliary atresia or a choledochal cyst. Thus a small cyst with anechoic content is highly suggestive of biliary atresia, whereas a cystic formation that increases in size is in favour of choledochal cyst.

Postnatally, ultrasound is the first-line examination with a specificity that varies between $71 \%$ and $97 \%$ [5]. The choledocal cyst appears as a cystic formation well- defined thin-walled (fig.1, 2, 3) sometimes containing an echogenic material corresponding to the Biliary Sludge (fig.4). It sits in contact with the portal vein and the gallbladder, communicating with the bile ducts which can be dilated [5].

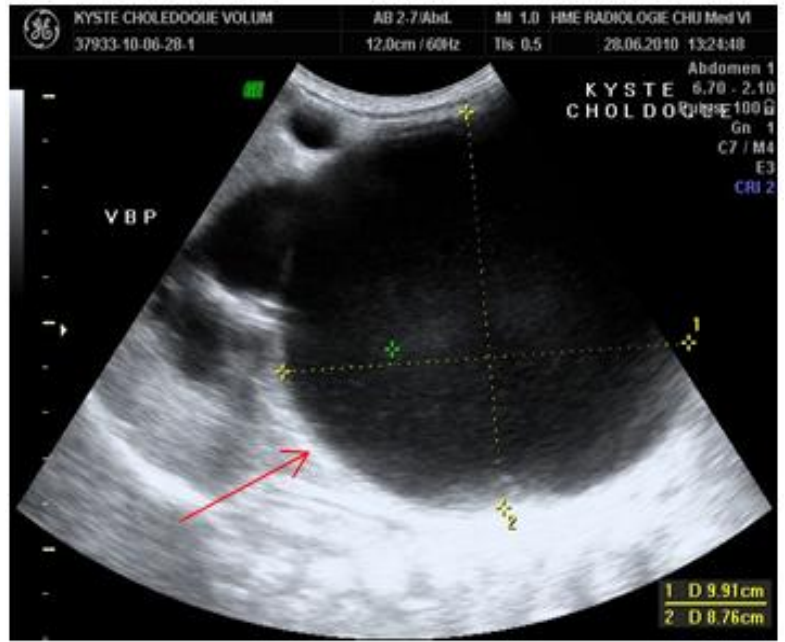

Fig-1: Hepato-biliary ultrasound showing a cystic formation in the Hepatic hilum continuing with the main bile duct: choledocal cyst type 1

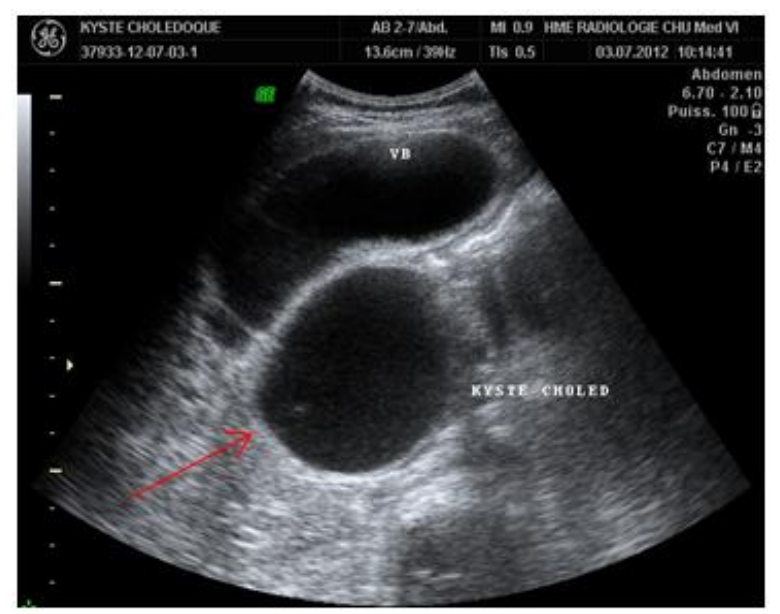

Fig-2: Abdominal ultrasound showing à cystic formation between the head of the pancreas and the gallbladder: choledocal cyst type 1

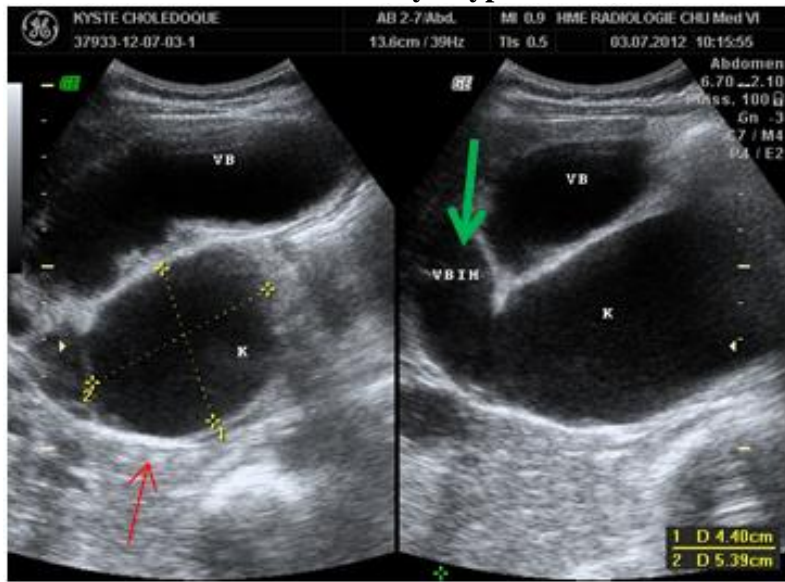

Fig-3: Hepato-biliary ultrasound showing the presence of cystic dilatation of the main biliary tract (red arrow) associated with dilation of the intrahepatic biliary ducts (green arrow) 


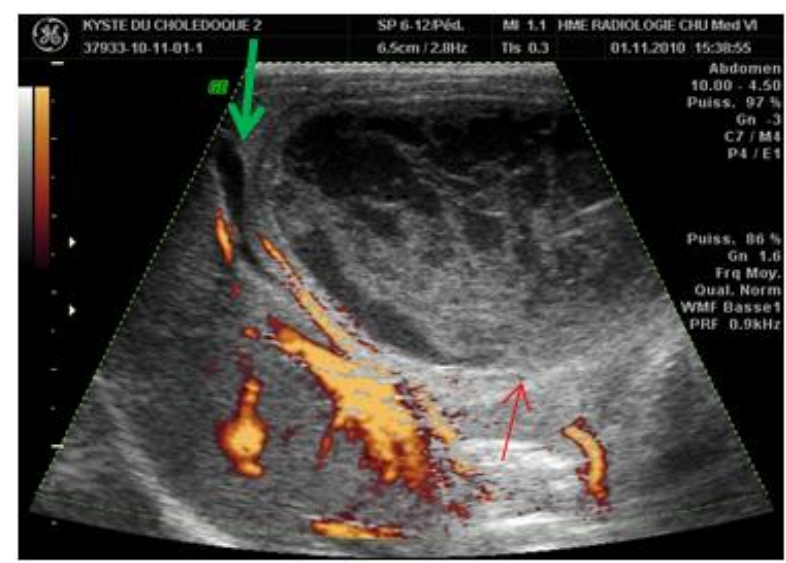

Fig-4: Hepatobiliary ultrasonography demonstrating a cystic formation of the hepatic hilus containing Sludge (red arrow) and compressing the Gallbladder (green arrow)

Ultrasound also allows the study of the state of the intrahepatic bile ducts and possibly can detect signs suggestive of a complication.

In ultrasonography, the differential diagnosis arises in the neonatal period with biliary atresia (cystic form), and in children and the young adult with a dilation of the bile ducts upstream of a choledocholithiasis or a cystic lesion of the head of the pancreas.

CT scan allows a good characterization of the cyst which appears as a mass of the hepatic hilum, in contact with the head of the pancreas, iso or hypo dense thin wall, unmodified after the injection of the contrast product. The CT also allows the study of the distal portion of the common biliary duct, the head of the pancreas, the gallbladder, and finally allows the study of possible complications (fig. 5).

The CT cholangiography allows visualizing the accumulation of the contrast medium, excreted in the bile duct, in the cyst and allows a precise mapping of the biliary tree [6].
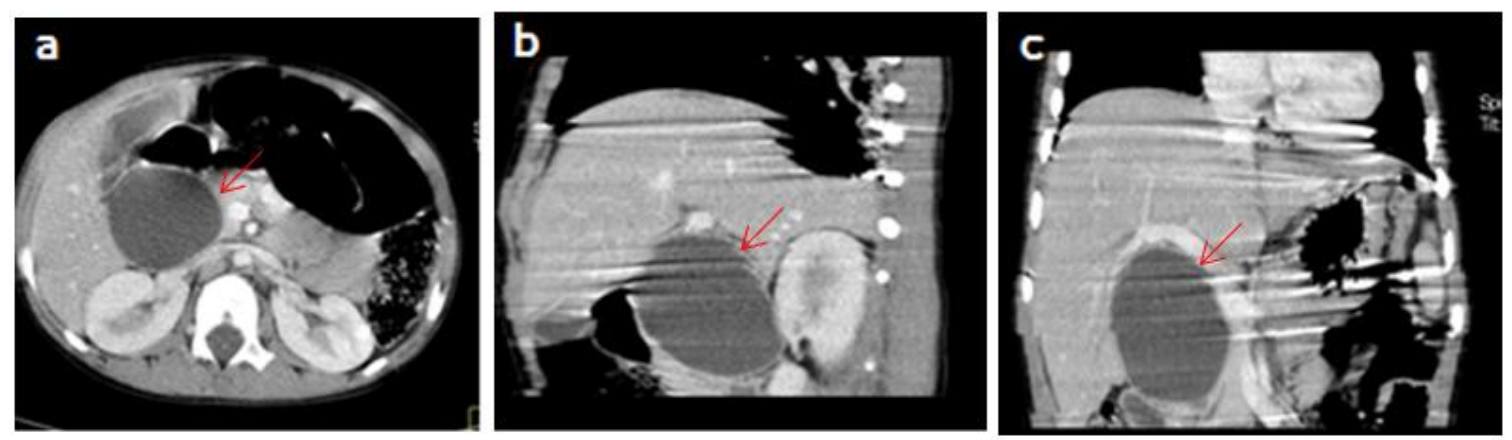

Fig-5: Abdominal CT with injection of contrast product: Axial view (a) -Sagittal view (b)- Coronal view (c). Supra-pancreatic Hilar hepatic mass, fluid content, with thin walls, not enhanced by contrast (red arrow). Choledochal cyst type I of Todani

Magnetic

Resonance

Cholangiopancreatography (MRCP), using heavily T2weighted sequences (HASTE sequences), is the best test to diagnose a choledochal cyst (fig. 6, 7, 8). It is a recent, non-invasive technique that allows a better analysis of the biliary and pancreatic ducts and biliopancreatic junction, as it makes it possible to rule out differential diagnoses $[7,8]$. 


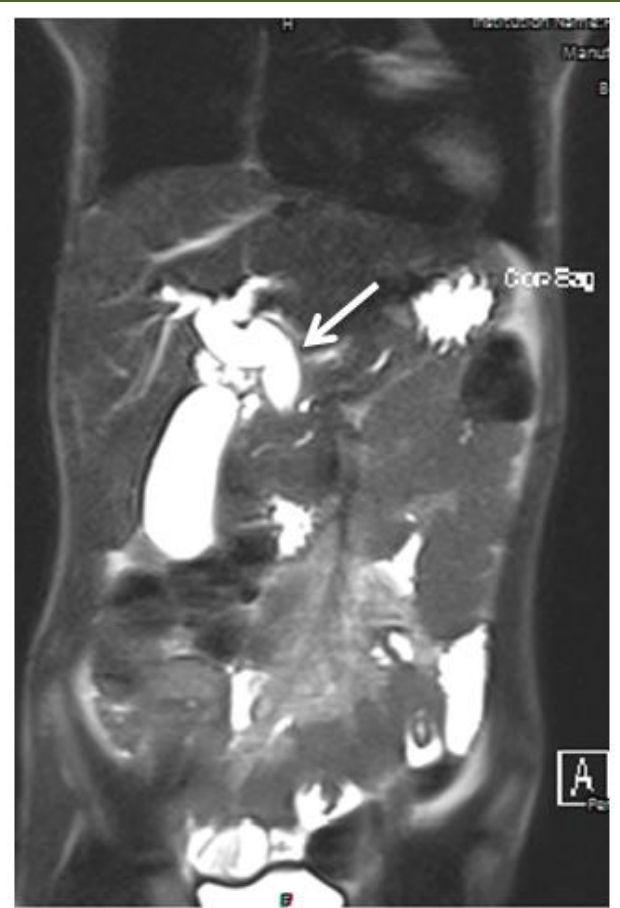

Fig-6: Coronal T2-weighted MRCP shows dilation of common bile duct (Arrow). Choledochal cyst type IA

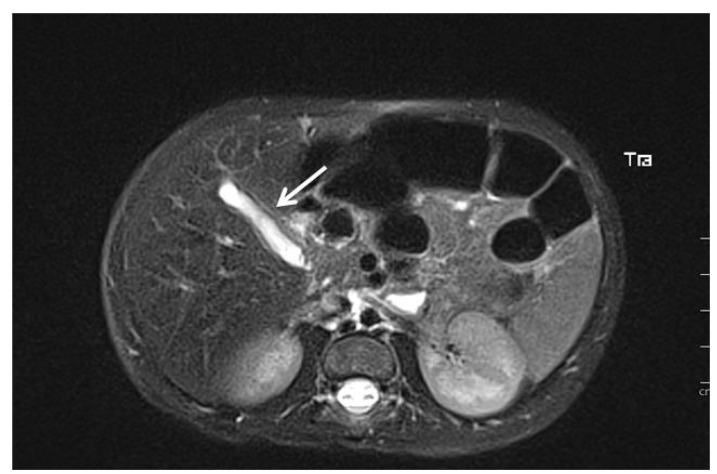

Fig-7: Axial T2-weighted MRCP shows a fusiform dilation of common bile duct( arrow). Choledochal cyst type Ic

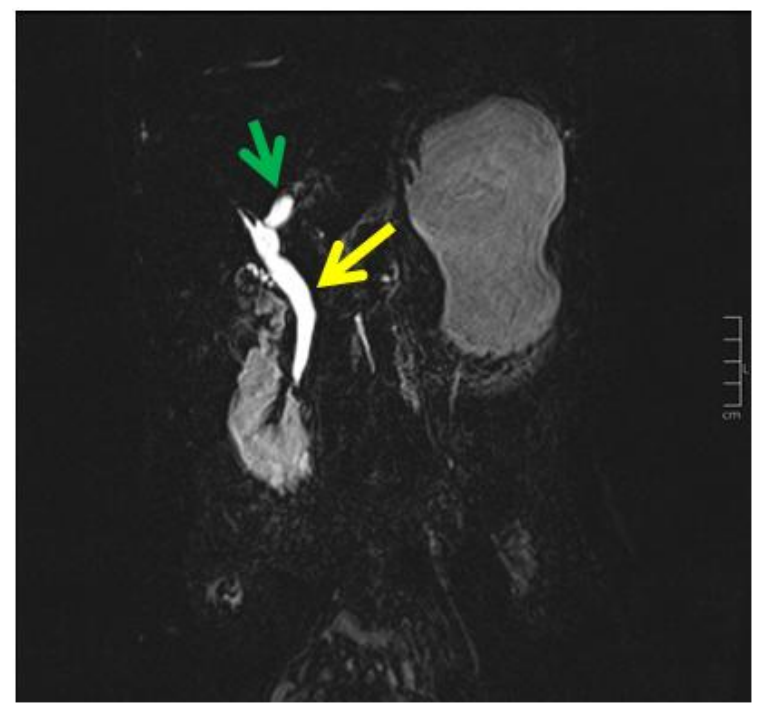

Fig-8: Coronal T2-weighted MRCP shows a cystic dilatation of the main biliary tract (yellow arrow) associated with dilation of the intrahepatic biliary ducts (green arrow). Bile duct cyst type IV?
The direct opacifications of the biliary system find their interest mainly peroperatively

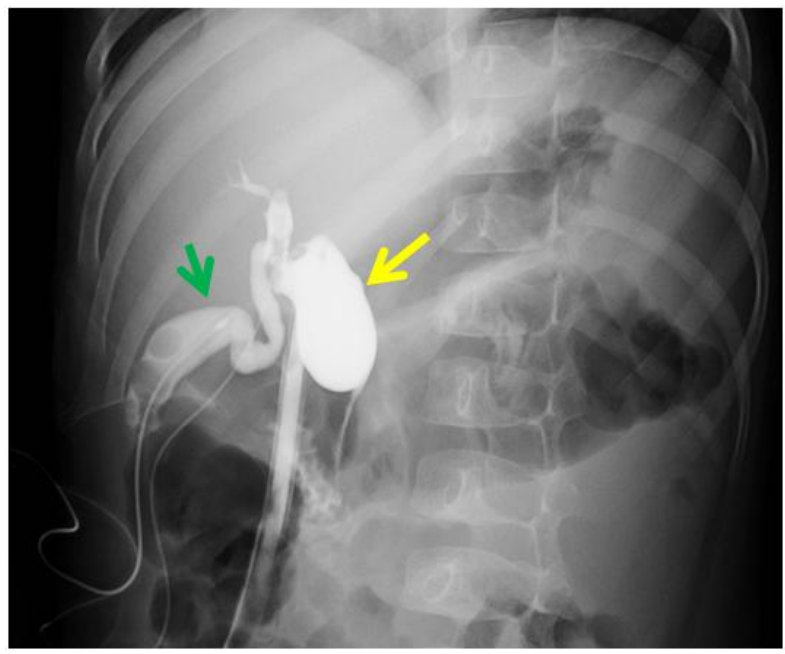

Fig-9: Intraoperative cholangiography showing a sacciform cystic dilatation of the common bile duct type 1b according to TODANI (yellow arrow) and the dilation of the cystic duct (green arrow)

The usual complications of choledochal cysts are stones that can block low bile duct, spontaneous rupture, pancreatitis, primary biliary cirrhosis, portal hypertension, cholangitis and malignant degeneration to a cholangiocarcinoma.

The treatment of this pathology is surgical and consists of a complete resection of the cyst with $y$ shapped hepatico- jejunal anastomosis and disconnection of the bile duct from the wirsung. This wide resection is indicated because of the risk of secondary degeneration of the dysplastic biliary wall.

\section{CONCLUSION}

The choledochal cyst is a malformation that can be diagnosed pre- and postnatally. Medical imaging, including initially ultrasound, and sectional imaging play a vital role in their diagnosis. The MRCP is the examination of choice that allows accurate biliary mapping, thus avoiding the use of invasive opacifications.

\section{REFERENCES}

1. Savic DJ, Milovanovic D, Jovanovic D. Congenital dilatation of the common bile duct (congenital choledochal cyst). Srp Arth Celok Lek. 2001; 129:47-50.

2. Babbitt DP, Starshak RJ, Clemett AR. Choledochal cyst: a concept of etiology. American Journal of Roentgenology. 1973 Sep;119(1):57-62.

3. Vullierme MP, Vilgrain V, Zins M, Sibert A, Denis A, Belghiti J. Dilatations kystiques congénitales de la voie biliaire principale. Gastroenterol Clin Biol. 1997;21:201-8. 
4. Dumitrascu T1, Lupescu I, Ionescu M. The Todani classification for bile duct cysts: an overview Acta Chir Belg. 2012 Sep-Oct; 112(5):340-5.

5. Metcalfe MS1, Wemyss-Holden SA, Maddern GJ. Management dilemmas with choledochal cysts. Arch Surg. 2003 Mar;138(3):333-9.

6. Akhan O, Demirkazik FB, Özmen MN, Ariyürek M. Choledochal cysts: ultrasonographic findings and correlation with other imaging modalities. Abdominal imaging. 1994 May;19(3):243-7.

7. Huang CT, Lee HC, Chen WT, Jiang CB, Shih SL, Yeung CY. Usefulness of magnetic resonance cholangiopancreatography in pancreatobiliary abnormalities in pediatric patients. Pediatrics \& Neonatology. 2011 Dec 1;52(6):332-6.

8. Miyazaki T, Yamashita Y, Tang Y, Tsuchigame T, Takahashi M, Sera Y. Single-shot MR cholangiopancreatography of neonates, infants, and young children. AJR. American journal of roentgenology. 1998 Jan;170(1):33-7.

9. Mannai S, Kraiem T, Gharbi L, Haoues N, Mestiri $\mathrm{H}$, Khalfallah M-T. les dilatations kystiques congénitales des voies biliaires. Annales de chirurgie. 2006; 131:369-374. 